\title{
PERIODIC SOLUTIONS OF QUASI-DIFFERENTIAL EQUATIONS
}

\author{
ABDELKADER BOUCHERIF \\ University of Tlemcen, Department of Mathematics \\ BP 119 Tlemcen, 13000, Algeria \\ EDUARDO GARCÍA-RÍO and JUAN J. NIETO ${ }^{1}$ \\ Universidad de Santiago de Compostela \\ Departamento de Análisis Matematico \\ Facultad de Matemáticas, Spain
}

(Received January, 1995; Revised December, 1995)

\begin{abstract}
Existence principles and theorems are established for the nonlinear problem $L u=f(t, u)$ where $L u=-\left(\rho u^{\prime}\right)^{\prime}+h u$ is a quasi-differential operator and $f$ is a Carathéodory function. We prove a maximum principle for the operator $L$ and then we show the validity of the upper and lower solution method as well as the monotone iterative technique.
\end{abstract}

Key words: Sturm-Liouville Problem, Periodic Solution, Maximum Principle, Upper and Lower Solution.

AMS (MOS) subject classifications: $34 \mathrm{~B} 15,34 \mathrm{C} 25$.

\section{Introduction}

The linear equation

$$
-\left(\rho(t) u^{\prime}(t)\right)^{\prime}+h(t) u(t)=0, \quad t \in \mathbb{R}
$$

is one of the most widely studied differential equations in the mathematical literature. In the mid-1800s, Sturm and Liouville observed that even for $\rho \equiv 1$ there is no closed form available in general, and they started a study of the qualitative properties of the solutions. On the other hand, it is obvious that one of the most relevant areas of research in the qualitative theory of differential equations is the study of existence of periodic solutions. Consequently we shall study the periodic boundary value problem for some linear and nonlinear equations related to the quasi-differential operator

$$
L u=-\left(\rho u^{\prime}\right)^{\prime}+h u .
$$

After imposing some conditions on $\rho$ and introducing the new independent variable $x=\int_{c}^{t} \frac{1}{\rho}$, so that (1.1) becomes

$$
-v^{\prime \prime}+H(x) v=0
$$

\footnotetext{
${ }^{1}$ Research partially supported by DGICY'T, projects PB91-0793 and PB94-0610. 
we study the operator $L$ in the form (1.2) since it is symmetric (formally self-adjoint) and many of the celebrated equations of mathematical physics appear as in (1.2). For the relation between these two forms for second and higher order differential equations, the reader is referred to [4] and [7].

We consider the following periodic boundary value problem

$$
\begin{gathered}
-\left(\rho u^{\prime}\right)^{\prime}+h u=f(t, u), \quad \text { for a.e. } t \in I=[0,2 \pi] \\
u(0)=u(2 \pi), \quad u^{\prime}(0)=u^{\prime}(2 \pi) .
\end{gathered}
$$

Throughout this paper, we shall refer to the nonlinear problem (1.4), (1.5) as (NP), and further, we shall assume that the coefficient functions $\rho$ and $h$ satisfy

(i) $\quad \rho \in W^{1,1}(I)$ (Sobolev space of first order), $\rho(t) \geq \rho_{0}>0, \rho(0)=\rho(2 \pi)$.

(ii) $h \in L^{1}(I), h \gtrsim 0$; that is, $h(t) \geq 0$ for a.e. $t \in I$ and $h(t)>0$ on a subset of $I$ of positive measure.

Also, the function $f: I \times \mathbb{R} \mapsto \mathbb{R}$ is assumed to satisfy the Caratheodory conditions; that is, $f(\cdot, u)$ is measurable for every $u \in \mathbb{R}, f(t, \cdot)$ is continuous for a.e. $t \in I$, and for every $R>0$ there exists a function $h_{R} \in L^{1}(I)$ with

$$
|f(t, u)| \leq h_{R}(t) \text { for a.e. } t \in I \text { and every }|u| \leq R .
$$

By a solution of (NP) we mean a function $u \in W^{2,1}(I)$ satisfying the equation for a.e. $t \in I$ and $u(0)=u(2 \pi), u^{\prime}(0)=u^{\prime}(2 \pi)$, where $W^{2,1}(I)$ denotes the Sobolev space of second order [3]. If $\rho \equiv 1$ and $h \equiv M>0$ then $L u=-u^{\prime \prime}+M u$ and we extend the well-known results for the existence of periodic solutions of $-u^{\prime \prime}=f(t, u)$ for $f$ continuous (see [5], [8]) and for $f$ a Carathéodory function (see [9]).

We first prove a maximum principle for the operator $L$ with periodic boundary conditions. In particular, we obtain that $L$ is invertible and that the corresponding Green's function is nonnegative. This allows us to give some existence results.

In section 3 we define the concept of upper solution, $\beta$, and lower solution, $\alpha$. If $\alpha \leq \beta$ then (NP) has at least one solution between $\alpha$ and $\beta$. In the study of (NP) the function $\Gamma(t)=$ $\limsup _{|u| \rightarrow \infty} \frac{f(t, u)}{u}$ plays an important role. In $\S 4$, we show that $h \gtrsim \Gamma$ implies that (NP) is solvable. Finally, under a one-sided Lipschitz condition, we show that validity of the monotone iterative method that approximates the minimal and maximal solutions between $\alpha$ and $\beta$.

\section{Preliminary Results}

In this section, we present some results concerning the linear problem

$$
\begin{gathered}
-\rho\left(u^{\prime}\right)^{\prime}+h u=\sigma \\
u(0)=u(2 \pi), \quad u^{\prime}(0)=u^{\prime}(2 \pi)
\end{gathered}
$$

where $\sigma \in L^{1}(I)$. We shall refer to problem (2.1), (2.2) as (LP).

Theorem 2.1: Assume $\sigma \in L^{1}(I)$ and $\sigma \gtrsim 0$. Then, any solution $u$ of (LP) is such that $u(t) \geq 0$ for a.e. $t \in I$.

Proof: Since $u$ is a solution of (LP), 


$$
-\int_{0}^{2 \pi}\left(\rho u^{\prime}\right)^{\prime}+\int_{0}^{2 \pi} h u=\int_{0}^{2 \pi} \sigma
$$

Thus, $\int_{0}^{2 \pi} h(t) u(t) d t=\int_{0}^{2 \pi} \sigma(t) d t$.

Now, if $u<0$ on $I$, then $h u \leq 0$ and hence $\int_{0}^{2 \pi} \sigma(t) d t \leq 0$, which is a contradiction with the condition on $\sigma$. Hence, $u\left(t_{M}\right)=\max \{u(t) ; t \in I\} \stackrel{0}{\geq} 0$. Let us show that the minimum of $u$ is nonnegative. Assume $u\left(t_{m}\right)=\min \{u(t) ; t \in I\}<0$. If $t_{m} \in(0,2 \pi)$ and $t_{m}<t_{M}$, let $t_{1} \in\left(t_{m}, t_{M}\right)$ with $u(t)<0$ on $\left(t_{m}, t_{1}\right), u\left(t_{1}\right)=0$. Then $h u \leq 0$ on $\left(t_{m}, t_{1}\right)$, and hence

$$
-\left(\rho u^{\prime}\right)^{\prime} \geq-\left(\rho u^{\prime}\right)^{\prime}+h u=\sigma, \text { a.e. on }\left(t_{m}, t_{1}\right)
$$

which shows that $\rho u^{\prime}$ is decreasing on $\left(t_{m}, t_{1}\right)$. Since $t_{m}$ is minimum of $u, u^{\prime}\left(t_{m}\right)=0$, and hence $\rho u^{\prime} \leq 0$ on $\left(t_{m}, t_{1}\right)$. Also, from $(i)$ it follows that $u^{\prime}(t) \leq 0$ on $\left(t_{m}, t_{1}\right)$, and thus $u(t)$ is decreasing on $\left(t_{m}, t_{1}\right)$, which is a contradiction. If $t_{m}>t_{M}$, let $t_{2} \in\left(t_{M}, t_{m}\right)$ with $u(t)>0, t \in\left(t_{2}, t_{m}\right)$, and $u\left(t_{2}\right)=0$. As before, we have that $\rho u^{\prime}$ is decreasing on $\left(t_{2}, t_{m}\right)$. Now, from $u^{\prime}\left(t_{m}\right)=0$ we get that $u^{\prime} \geq 0$ on $\left(t_{2}, t_{m}\right)$ and that $u$ is increasing on $\left(t_{2}, t_{m}\right)$, which is again a contradiction.

Next, assume the minimum to be attained at $t_{m}=0$. Then $u^{\prime}(0) \geq 0$. It follows that $t=2 \pi$ is also minimum, and hence $u^{\prime}(2 \pi) \leq 0$ and $u^{\prime}(0)=0$. The proof follows as in the previous case.

Remark 2.1: The conclusion of the previous theorem is still valid if we replace the periodic conditions by $u(0)=u(2 \pi), u^{\prime}(0) \leq u^{\prime}(2 \pi)$.

Corollary 2.1: (LP) (with $\sigma=0$ ) has only the trivial solution.

Proof: Multiplying (2.1) by $u$ and integrating over $[0,2 \pi]$ we get

Thus, $u=0$.

$$
\int_{0}^{2 \pi}\left[\rho(s)\left(u^{\prime}(s)\right)^{2}+h(s) u^{2}(s)\right] d s=0 .
$$

Corollary 2.2: (LP) has a unique solution $u$, given by

$$
u(t)=\int_{0}^{2 \pi} G(t, s) \sigma(s) d s
$$

where $G(t, s)$ is the Green's function corresponding to (LP) for $\sigma=0$.

Proof: It is enough to construct the Green's function $G(t, s)$. Write $(2.1)$ in the following form

$$
u^{\prime \prime}(t)+a(t) u^{\prime}(t)+b(t) u(t)=c(t)
$$

where $a(t)=\rho^{\prime}(t) / \rho(t), b(t)=-h(t) / \rho(t)$ and $c(t)=-\sigma(t) / \rho(t)$.

Let $u_{1}, u_{2}$ be two linearly independent solutions of the homogeneous equation (2.1) with $\sigma=0$. Then, the general solution of (2.1) is given by

$$
u(t)=c_{1} u_{1}(t)+c_{2} u_{2}(t)+\int_{0}^{2 \pi} \frac{-u_{1}(t) u_{2}(s)+u_{2}(t) u_{1}(s)}{W(s)} c(s) d s
$$

where $W$ is the Wronskian of $u_{1}$ and $u_{2}$. The derivative of $u$ is given by 


$$
u^{\prime}(t)=c_{1} u_{1}^{\prime}(t)+c_{2} u_{2}^{\prime}(t)+\int_{0}^{2 \pi} \frac{-u_{1}^{\prime}(t) u_{2}(s)+u_{2}^{\prime}(t) u_{1}(s)}{W(s)} c(s) d s .
$$

To determine $c_{1}$ and $c_{2}$, we use (2.2) and get the following system

where

$$
\left(\begin{array}{cc}
u_{1}(0)-u_{1}(2 \pi) & u_{2}(0)-u_{2}(2 \pi) \\
u_{1}^{\prime}(0)-u_{1}^{\prime}(2 \pi) & u_{2}^{\prime}(0)-u_{2}^{\prime}(2 \pi)
\end{array}\right)\left(\begin{array}{c}
c_{1} \\
c_{2}
\end{array}\right)=\left(\begin{array}{c}
d_{1} \\
d_{2}
\end{array}\right)
$$

$$
\begin{aligned}
& d_{1}=\int_{0}^{2 \pi} \frac{-u_{1}(2 \pi) u_{2}(s)+u_{2}(2 \pi) u_{1}(s)}{W(s)} c(s) d s \\
& d_{2}=\int_{0}^{2 \pi} \frac{-u_{1}^{\prime}(2 \pi) u_{2}(s)+u_{2}^{\prime}(2 \pi) u_{1}(s)}{W(s)} c(s) d s .
\end{aligned}
$$

It follows from Corollary 2.1 that the above system has a unique solution

$$
\left(\begin{array}{c}
c_{1} \\
c_{2}
\end{array}\right)=\left(\begin{array}{ll}
u_{1}(0)-u_{1}(2 \pi) & u_{2}(0)-u_{2}(2 \pi) \\
u_{1}^{\prime}(0)-u_{1}^{\prime}(2 \pi) & u_{2}^{\prime}(0)-u_{2}^{\prime}(2 \pi)
\end{array}\right)^{-1}\left(\begin{array}{c}
d_{1} \\
d_{2}
\end{array}\right)
$$

Substituting the values of $c_{1}$ and $c_{2}$ into (2.4) we obtain the unique solution $u(t)$ of (LP). Moreover, $u(t)$ has the form $\int_{0}^{2 \pi} G(t, s) \sigma(s) d s$, with a uniquely determined function $G(t, s)$, which satisfies all the properties of the Green's function.

Lemma 2.1: Let $\gamma \in L^{1}(I)$ with $0 \gtrsim \gamma$. Then there exists $\delta(\gamma)=\delta>0$ such that

$$
B_{\gamma}(u)=B(u)=\int_{0}^{2 \pi}\left\{\rho(t)\left[u^{\prime}(t)\right]^{2}-\gamma(t) u^{2}(t)\right\} d t \geq \delta\|u\|_{H^{1}(I)}^{2},
$$

where $H^{1}(I)$ denotes $W^{2,1}(I)[3]$.

Proof: In $L^{2}(I)$ consider the following inner product

$$
(u, v)_{\rho}=\int_{0}^{2 \pi} \rho(t) u(t) v(t) d t
$$

which is equivalent to the usual one. We first show that $B(u)=0$ if and only if $u=0$. Obviously $u=0$ implies $B(u)=0$. Let $B(u)=0$. Thus $u$ is constant on $I$ since $B(u) \geq \int_{0}^{2 \pi} \rho(t)\left[u^{\prime}(t)\right]^{2} d t$. Consequently, $0=u^{2} \int_{0}^{2 \pi} \gamma(t) d t$ and $u=0$.

If the conclusion of the lemma is not true, then there exists a sequence $\left\{v_{n}\right\}$ in $H^{1}(I)$ such that $B\left(v_{n}\right)<\frac{1}{n}\left\|v_{n}\right\|_{H^{1}(I)}^{2}$. Let $u_{n}=v_{n} /\left\|v_{n}\right\|_{H^{1}(I)}$. Thus (passing to a subsequence) we have that $\left\{B\left(u_{n}\right)\right\} \rightarrow 0,\left\{u_{n}\right\} \rightarrow u$ in $C(\bar{I}),\left\{u_{n}\right\} \rightarrow u$ (weak) in $H^{1}(I)$ and $\left\{u_{n}^{\prime}\right\} \rightarrow u^{\prime}$ (weak) in $L^{2}(I)$.

Thus $\int_{0}^{2 \pi} \rho(t)\left[u^{\prime}(t)\right]^{2} d t \leq \liminf _{n \rightarrow \infty} \int_{0}^{2 \pi} \rho(t)\left[u_{n}^{\prime}(t)\right]^{2} d t$ and 


$$
\lim _{n \rightarrow \infty} \int_{0}^{2 \pi} \rho(t)\left[u_{n}^{\prime}(t)\right]^{2} d t=\int_{0}^{2 \pi}-\gamma(t) u^{2}(t) d t
$$

Hence, $B(u) \leq 0$ and $u=0$ which implies that $\int_{0}^{2 \pi} \rho(t)\left[u_{n}^{\prime}(t)\right]^{2} d t=0$ and $\int_{0}^{2 \pi} u_{n}^{2}(t) d t=0$. From this we obtain that $\lim _{n \rightarrow \infty}\left\|u_{n}\right\|_{H^{1}(I)}=0$ which is a contradiction.

Remark 2.2: If $\gamma_{1}, \gamma_{2} \in L^{1}(I)$ satisfy $0 \gtrsim \gamma_{2} \geq \gamma_{1}$, then we can take $\delta\left(\gamma_{1}\right) \geq \delta\left(\gamma_{2}\right)>0$ since

$$
B_{\gamma_{1}}(u)=\int_{0}^{2 \pi}\left[\rho\left(u^{\prime}\right)^{2}-\gamma_{1} u^{2}\right] \geq \int_{0}^{2 \pi}\left[\rho\left(u^{\prime}\right)^{2}-\gamma_{2} u^{2}\right] \geq \delta\left(\gamma_{2}\right)\|u\|_{H^{1}(I)} .
$$

Now, let $W=\left\{u \in W^{2,1}(I) ; u(0)-u(2 \pi)=u^{\prime}(0)-u^{\prime}(2 \pi)=0\right\}$. From corollaries 2.1 and 2.2 we have that $L: W \mapsto L^{1}(I)$ is invertible and $L^{-1}$ is the integral operator whose kernel is $G$.

We end this section with the following result concerning (NP).

Theorem 2.2: Suppose $f: I \times \mathbb{R} \mapsto \mathbb{R}$ is a bounded function in the Carathéodory class. Then (NP) has at least one solution.

Proof: Let $N: L^{1}(I) \mapsto L^{1}(I)$ be defined by $(N u)(t)=f(t, u(t))$. Since $f$ is bounded, there exists a constant $c_{0}$ such that

$$
|f(t, u)| \leq c_{0}, \quad \forall(t, u) \in I \times \mathbb{R} .
$$

This implies that $N$ maps $L^{1}(I)$ into the closed ball of radius $2 \pi c_{0}$ in $L^{1}(I)$. Consequently (see [1, Ch. 3]), $N$ is continuous. Also, (NP) is equivalent to the fixed point problem

$$
u=T u
$$

where $T=i \circ L^{-1} \circ N, i: L^{1}(I) \mapsto L^{1}(I)$ being the inclusion.

Note that there exists a constant $c>0$ such that $\|T u\| \leq c$ for all $u \in L^{1}(I)$. Therefore, all solutions of $u=\lambda T u, \lambda \in[0,1]$ are bounded. This implies that $T$ has a fixed point [11, Th. 4.3.2], and hence, problem (NP) has at least one solution.

\section{Upper and Lower Solutions}

We say that $\alpha \in W^{2,1}(I)$ is a lower solution of (NP), if

$$
\left\{\begin{array}{c}
-\left(\rho \alpha^{\prime}\right)^{\prime}+h \alpha \leq f(t, \alpha), \quad \text { a.e } t \in I \\
\alpha(0)=\alpha(2 \pi) \\
\alpha^{\prime}(0) \geq \alpha^{\prime}(2 \pi) .
\end{array}\right.
$$

Similarly, we say that $\beta \in W^{2,1}(I)$ is an upper solution of (NP), if

$$
\left\{\begin{array}{c}
-\left(\rho \beta^{\prime}\right)^{\prime}+h \beta \geq f(t, \beta), \quad \text { a.e } t \in I \\
\beta(0)=\beta(2 \pi) \\
\beta^{\prime}(0) \leq \beta^{\prime}(2 \pi) .
\end{array}\right.
$$

Next, we will show that the existence of lower and upper solutions with $\alpha \leq \beta$ is a sufficient 
condition to guarantee the existence of solution of (NP) in the sector $[\alpha, \beta]=\left\{u \in W^{2,1}(I)\right.$; $\alpha(t) \leq u(t) \leq \beta(t), \forall t\})$. Relative to $\alpha, \beta$ consider the following functions $p(t, u)=\max \{\alpha(t)$, $\min \{u, \beta(t)\}\}, F(t, u)=f(t, p(t, u))$, and the modified problem

$$
\left\{\begin{array}{c}
-\left(\rho u^{\prime}\right)^{\prime}+h u=F(t, u) \\
u(0)=u(2 \pi), u^{\prime}(0)=u^{\prime}(2 \pi) .
\end{array}\right.
$$

Since $F(t, u)$ is a Carathéodory function on $I \times \mathbb{R}$, and moreover, it is bounded, it follows from Theorem 2.2 that (3.3) has a solution.

Theorem 3.1: Suppose that $\alpha, \beta$ are lower and upper solutions for (1.4), (1.5) respectively, such that $\alpha(t) \leq \beta(t)$ for every $t \in I$. Then there exists at least one solution $u$ of $(\mathrm{NP})$ such that $\alpha(t) \leq u(t) \leq \beta(t)$ for every $t \in I$.

Proof: It is clear that the modified problem (3.3) coincides with (NP) in the sector $[\alpha, \beta]$. Hence, it will suffice to show that any solution of (3.3) lies between $\alpha$ and $\beta$. Let us show that $\alpha \leq u$. (That $u \leq \beta$ is obtained in an analogous way.)

$\int_{0}^{2 \pi}$ Let us suppose that $u(t)<\alpha(t), \forall t \in I$. Since $-\left(\rho u^{\prime}\right)^{\prime}+h u=F(t, u),-\int_{0}^{2 \pi}\left(\rho u^{\prime}\right)^{\prime}+\int_{0}^{2 \pi} h u=$, and using the properties of $\rho$ and $(3.3)$,

$$
\begin{aligned}
0 & =\int_{0}^{2 \pi}[f(s, \alpha(s))-h(s) u(s)] d s \\
& \geq \int_{0}^{2 \pi} h(s)[\alpha(s)-u(s)] d s>0
\end{aligned}
$$

which is a contradiction. Hence, there exist points $t^{\prime} \in I$ such that $\alpha\left(t^{\prime}\right) \leq u\left(t^{\prime}\right)$.

Consider the function $\varphi(t)=\alpha(t)-u(t)$ and take $t_{0} \in I$ with $\varphi\left(t_{0}\right)=\max \{\varphi(t) ; t \in I\}$. Next assume that $\varphi\left(t_{0}\right)>0$ and take $t_{1} \in I$ with $\varphi\left(t_{1}\right) \leq 0$. If $t_{0} \in(0,2 \pi)$ and $t_{0}<t_{1}$, take $t_{2} \in\left(t_{0}, t_{1}\right)$ such that $\varphi\left(t_{2}\right)=0, \varphi(t)>0$ for all $t \in\left[t_{0}, t_{2}\right)$.

Since $-\left(\rho \alpha^{\prime}\right)^{\prime}+h \alpha \leq f(t, \alpha)$ and $p(t, u)=\alpha$ in $\left[t_{0}, t_{2}\right),-\left(\rho u^{\prime}\right)^{\prime}+h u=f(t, \alpha)$, and hence, $\rho \varphi^{\prime}$ is increasing on $\left[t_{0}, t_{2}\right)$. Since $\varphi^{\prime}\left(t_{0}\right)=0, \varphi^{\prime}(t) \geq 0, \forall t \in\left[t_{0}, t_{2}\right)$. This shows that $\varphi$ is increasing in $\left[t_{0}, t_{2}\right)$, which is a contradiction. If $t_{0}>t_{1}$, the result will be obtained in the same way (see the proof of Theorem 2.1).

Next, if $\varphi$ attains its maximum at $t_{0}=0, \varphi^{\prime}(0) \leq 0$. Since $t=2 \pi$ is also maximum, $\varphi^{\prime}(2 \pi) \geq 0$. Now, since $\varphi^{\prime}(0) \geq \varphi^{\prime}(2 \pi), \varphi^{\prime}(0)$ must equal 0 , and the result follows as in the previous case.

If $\alpha$ is a constant lower solution then $\alpha$ must satisfy

$$
h(t) \alpha \leq f(t, \alpha) \text { for a.e. } t \in \in I .
$$

Analogously, if $\beta$ is a constant upper solution, then $h(t) \beta \geq f(t, \beta)$ for a.e. $t \in I$. Thus we obtain the following corollary.

Corollary 3.1: Assume that

$$
\frac{f(t, u)}{u} \leq h(t) \text { for }|u| \text { sufficiently large and a.e. } t \in I \text {. }
$$


Then (NP) has at least one solution.

Proof: Let $R>0$ be such that $\frac{f(t, R)}{R} \leq h(t)$ for a.e. $t \in I$. Hence $\beta(t)=R, t \in I$ is an upper solution. Similarly $\alpha(t)=r<0, t \in I$ with $\frac{f(t, r)}{r} \leq h(t)$ for a.e. $t \in I$ is a lower solution.

\section{Existence Results}

We first consider the problem

$$
\begin{gathered}
-\left(\rho u^{\prime}\right)^{\prime}+h u=c u+\sigma \\
u(0)=u(2 \pi), \quad u^{\prime}(0)=u^{\prime}(2 \pi)
\end{gathered}
$$

where $c, \sigma \in L^{1}(I)$.

If $h \gtrsim c$ on $I$ then by Corollary $2.2(4.1),(4.2)$ has exactly one solution. To extend this result to (NP) we need some "a priori" estimates for the solutions of the nonlinear problem.

Lemma 4.1: Suppose that there exists $\gamma \in L^{1}(I)$ with $0 \gtrsim \gamma$ and $\epsilon \in[0, \delta)$, where $\delta$ is defined by Lemma 2.1, with

$$
c(t) \leq h(t)+\gamma(t)+\epsilon \text { for a.e. } t \in I \text {. }
$$

Then there exists $a>0$ such that

$$
\|u\|_{H^{1}(I)} \leq a(\delta-\epsilon)^{-1}\|\sigma\|_{L^{1}}
$$

for any solution of (4.1), (4.2).

Proof: Multiply (4.1) by $u$ and integrate on $I$ to get

$$
\int_{0}^{2 \pi} \rho(t)\left[u^{\prime}(t)\right]^{2} d t+\int_{0}^{2 \pi}[h(t)-c(t)] u(t)^{2} d t=\int_{0}^{2 \pi} \sigma(t) u(t) d t .
$$

Hence,

$$
\begin{gathered}
\int_{0}^{2 \pi} \sigma(t) u(t) d t \geq \int_{0}^{2 \pi}\left\{\rho(t)\left[u^{\prime}(t)\right]^{2}-\gamma(t) u(t)^{2}\right\} d t-\epsilon \int_{0}^{2 \pi} u^{2}(t) d t \\
=B_{\gamma}(u)-\epsilon\|u\|_{L^{2}(I)}^{2} \\
\geq \delta(\gamma)\|u\|_{H^{1}(I)}^{2}-\epsilon\|u\|_{L^{2}(I)}^{2} \\
\geq(\delta-\epsilon)\|u\|_{H^{1}(I)}^{2}
\end{gathered}
$$

On the other hand,

$$
\int_{0}^{2 \pi} \sigma(t) u(t) d t \leq\|\sigma\|_{L^{1}(I)} \cdot\|u\|_{L^{\infty}(I)} \leq a\|\sigma\|_{L^{1}(I)} \cdot\|u\|_{H^{1}(I)} .
$$

In consequence, $\|u\|_{H^{1}(I)} \leq a(\delta-\epsilon)^{-1}\|\sigma\|_{L^{1}(I)}$.

Theorem 4.1: Suppose that there exists $\gamma \in L^{1}(I)$ with $0 \gtrsim \gamma$ and 


$$
h(t)+\gamma(t) \geq \Gamma(t)=\limsup _{|u| \rightarrow \infty} \frac{f(t, u)}{u}
$$

uniformly for a.e. $t \in I$. Then the nonlinear problem (NP) has at least one solution.

Proof: There exists $M>0$ such that $|u| \geq M$ implies

$$
\frac{f(t, u)}{u} \leq h(t)+\gamma(t)+\frac{\delta}{2}, \quad \text { for a.e. } t \in I .
$$

Define

$$
g(t, u)=\left\{\begin{array}{cc}
\frac{f(t, u)}{u} & |u| \geq M \\
\frac{f(t, M)}{M} & 0<u<M \\
\frac{f(t,-M)}{-M} & -M<u<0 \\
h(t)+\gamma(t) & u=0
\end{array}\right.
$$

and $d(t, u)=f(t, u)-g(t, u) u$.

It is easy to see that $g$ and $d$ are Carathéodory functions such that

$$
g(t, u) \leq h(t)+\gamma(t)+\frac{\delta}{2}, \text { for } u \in \mathbb{R} \text { and a.e. } t \in I
$$

and

$$
|d(t, u)| \leq \sup _{|u| \leq M}|f(t, u)-g(t, u) u| \leq 2\left|h_{M}(t)\right| .
$$

Thus, (NP) is equivalent to

$$
\begin{gathered}
-\left(\rho u^{\prime}\right)^{\prime}+h u=d(t, u)+g(t, u) u \\
u(0)=u(2 \pi), \quad u^{\prime}(0)=u^{\prime}(2 \pi) .
\end{gathered}
$$

This suggests to consider the following homotopy:

$$
\begin{gathered}
-\left(\rho u^{\prime}\right)^{\prime}+h u=[(1-\lambda) \gamma+(1-\lambda) h+\lambda g(t, u)] u+\lambda d(t, u), \\
u(0)=u(2 \pi), \quad u^{\prime}(0)=u^{\prime}(2 \pi) .
\end{gathered}
$$

Equation (4.3) is equivalent to

$$
-\left(\rho u^{\prime}\right)^{\prime}-\gamma u=\lambda[-\gamma u-h u+g(t, u) u+d(t, u)] .
$$

If $L_{\gamma}: W^{2,1}(I) \mapsto L^{1}(I)$ is defined by $L_{\gamma} u=-\left(\rho u^{\prime}\right)^{\prime}-\gamma u$ with $-\gamma \gtrsim 0$, then the set of equations $(4.3),(4.4)$ is equivalent to

$$
u=\lambda\left(L_{\gamma}^{-1} \circ N\right) u
$$

where $N u=-\gamma u-h u+g(t, u) u+d(t, u)$.

On the other hand, (4.3) is of the form (4.1) with

$$
c(t)=(1-\lambda) \gamma(t)+(1-\lambda) h(t)+\lambda g(t, u(t))
$$


and $\sigma(t)=\lambda d(t, u(t))$. We have that

$$
\begin{gathered}
c(t) \leq(1-\lambda)[h(t)+\gamma(t)]+\lambda[h(t)+\gamma(t)]+\lambda \frac{\delta}{2} \\
\leq h(t)+\gamma(t)+\frac{\delta}{2},
\end{gathered}
$$

and $|\lambda d(t, u(t))| \leq 2 h_{M}(t)$ for a.e. $t \in I$, which implies that $\|\sigma\|_{L^{1}(I)} \leq 2\left\|h_{M}\right\|_{L^{1}(I)}$.

Consequently,

$$
\|u\|_{H^{1}(I)} \leq a\left(\frac{\delta}{2}\right)^{-1} 2\left\|h_{M}\right\|_{L^{1}(I)}=4 a \delta^{-1}\left\|h_{M}\right\|_{L^{1}(I)}
$$

and all the solutions of (4.3), (4.4) are bounded independently of $\lambda \in(0,1)$.

In consequence, by [11, Th. 4.3.2], (4.6) has a fixed point for $\lambda=1$ which is a solution of (NP).

\section{The Monotone Method}

In this section we assume that $\alpha$ is a lower solution and $\beta$ is an upper solution of (NP) respectively, such that $\alpha \leq \beta$ on $I$.

We introduce the following additional hypothesis: There exists $M \in L^{1}(I)$ with $M(t) \geq 0$ for a.e. $t \in I$, and

$$
f(t, u)-f(t, v) \geq-M(t)(u-v), \alpha(t) \leq v \leq u \leq \beta(t) .
$$

Next, for each $\eta \in L^{1}(I)$, consider the modified problem

$$
\left\{\begin{array}{c}
(L u)(t)+M(t) u(t)=f(t, \eta(t))+M(t) \eta(t) \equiv \sigma_{\eta}(t) \\
u(0)=u(2 \pi), \quad u^{\prime}(0)=u^{\prime}(2 \pi) .
\end{array}\right.
$$

Note that, since $\sigma_{\eta} \in L^{1}(I)$ and $M(t) \geq 0$ for a.e. $t \in I$, then (5.2) has a unique solution since $h+M \gtrsim 0$. Define an operator $A: L^{1}(I) \mapsto E$ by $A \eta=u$ to be the solution of (5.2). The basic properties of $A$ are summarized in the following.

Lemma 5.1: Assume that (5.1) holds. Then the operator $A$ has the following properties.

1. If $\eta \in L^{1}(I)$ is such that $\alpha \leq \eta \leq \beta$ for a.e. $t \in I$, then

$$
\alpha \leq A \eta \leq \beta \text {, for a.e. } t \in I \text {. }
$$

2. If $\eta_{1}, \eta_{2} \in L^{1}(I)$ are such that $\alpha \leq \eta_{1} \leq \eta_{2} \leq \beta$ for a.e. $t \in I$, then

$$
\alpha \leq A \eta_{1} \leq A \eta_{2} \leq \beta, \text { for a.e. } t \in I \text {. }
$$

Proof: Let us show that $\alpha \leq A \eta$ for a.e. $t \in I$. (The case $A \eta \leq \beta$ can be proved similarly.)

Let $v=u-\alpha$, where $u=A \eta$. From (5.1) it follows that $(L v)(t)+M(t) v(t) \geq 0$ for a.e. $t \in I$. Moreover, $v(0)=v(2 \pi)$ and $v^{\prime}(0) \leq v^{\prime}(2 \pi)$. Also note that $h+M \gtrsim 0$. Hence $v \geq 0$ for a.e. $t \in I$ as a consequence of Remark 2.1, and thus $\alpha \leq u=A \eta$.

To prove the second part, put $v=u_{2}-u_{1}$, where $u_{i}=A \eta_{i},(i=1,2)$. Then $(L v)(t)+$ $M(t) v(t) \geq 0$ for a.e. $t \in I$, and the result follows as before. 
Theorem 5.1: Suppose that $\alpha$ and $\beta$ are lower and upper solutions of (1.4), (1.5) respectively, such that $\alpha \leq \beta$. Assume that condition (5.1) holds. Then there exists monotone sequences $\left\{\alpha_{n}\right\}$, $\left\{\beta_{m}\right\}$ such that

1. $\left\{\alpha_{n}\right\}$ is increasing and $\left\{\beta_{m}\right\}$ is decreasing.

2. $\quad \alpha_{n} \leq \beta_{m}$ for any $n, m \in \mathbb{N}$.

3. $\quad \underset{\sim}{\tilde{\alpha}}=\lim \alpha_{n}$ is the minimal solution of $(\mathrm{NP})$ in $[\alpha, \beta]$.

4. $\tilde{\beta}=\lim \beta_{m}^{n}$ is the maximal solution of $(\mathrm{NP})$ in $[\alpha, \beta]$.

Moreover, if $u$ is a solution of (NP) with $u \in[\alpha, \beta]$, then $u \in[\widetilde{\alpha}, \widetilde{\beta}]$.

Proof: Let $\alpha_{0}=\alpha$ and define $\left\{\alpha_{n}\right\}$ inductively by $\alpha_{n+1}=A \alpha_{n}$, for $n \geq 0$. Similarly, let $\beta_{0}=\beta$ and $\beta_{m+1}=A \beta_{m}$ for $m \geq 0$. It follows now from the previous lemma that $\left\{\alpha_{n}\right\}$ is increasing and $\left\{\beta_{m}\right\}$ is decreasing. Also, $\alpha_{n} \leq \beta_{m}$ for all $n, m \in \mathbb{N}$.

Therefore, $\left\{\alpha_{n}\right\}$ is uniformly bounded and increasing, and it has a pointwise limit, say $\tilde{\alpha}(t)$, $t \in I$. Using the integral representation for $\alpha_{n+1}$ given by (2.3) and by standard arguments, it can be shown that $\tilde{\alpha}$ is a solution of (NP) and properties 1-4 are valid.

\section{References}

[1] Appell, J. and Zabrejko, P.P., Nonlinear Superposition Operators, Cambridge University Press, Cambridge 1990.

[2] Boucherif, A., Periodic boundary value problems with Carathéodory nonlinearities, Proc. Dynam. Systems Appl. 1 (1994), 25-32.

[3] Brezis, H., Analyse Fonctionnelle, Theorie et Applications, Masson, Paris 1983.

[4] Everitt, W.N. and Race, D., Some remarks on linear ordinary quasi-differential expressions, Proc. London Math. Soc. 54 (1987), 300-320.

[5] Ladde, G.S., Lakshmikantham, V. and Vatsala, A.S., Monotone Iterative Techniques for Nonlinear Differential Equations, Pitman, Boston 1985.

[6] Mawhin, J., Compactié, monotonie et convexité dans l'étude de problémes aux limites semi-linéares, Séminaire d'Analyse moderne 19, Université de Sherbrook (1981).

[7] Neuman, F., Global Properties of Linear Ordinary Differential Equations, Kluwer Academic Publishers, Dordrecht 1991.

[8] Nieto, J.J., Nonlinear second order periodic boundary value problems, J. Math. Anal. Appl. 130 (1988), 22-29.

[9] Nieto, J.J., Nonlinear second order periodic boundary problems with Carathéodory functions, Appl. Anal. 34 (1989), 111-128.

[10] Nieto, J.J. and Cabada, A., A generalized upper and lower solutions method for nonlinear second order ordinary differential equations, J. Applied Math. Stoch. Anal. 5 (1992), 157165.

[11] Smart, D.R., Fixed Point Theorems, Cambridge Univ. Press, Cambridge 1980. 


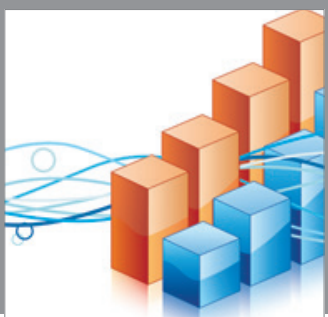

Advances in

Operations Research

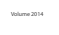

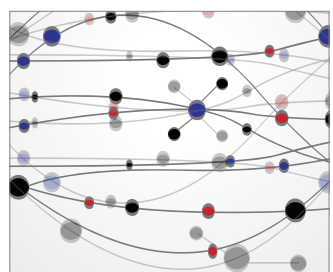

\section{The Scientific} World Journal
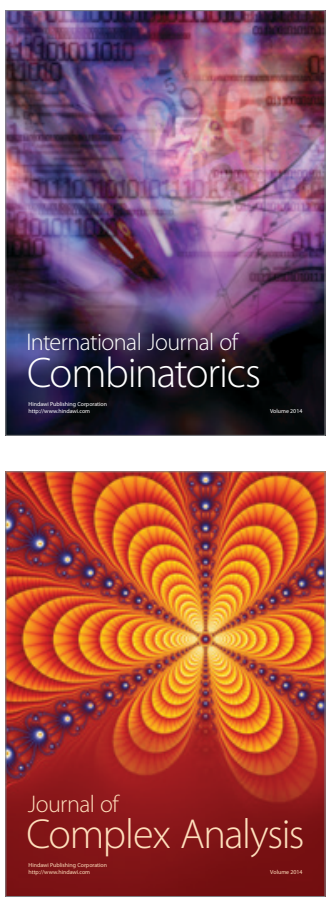

International Journal of

Mathematics and

Mathematical

Sciences
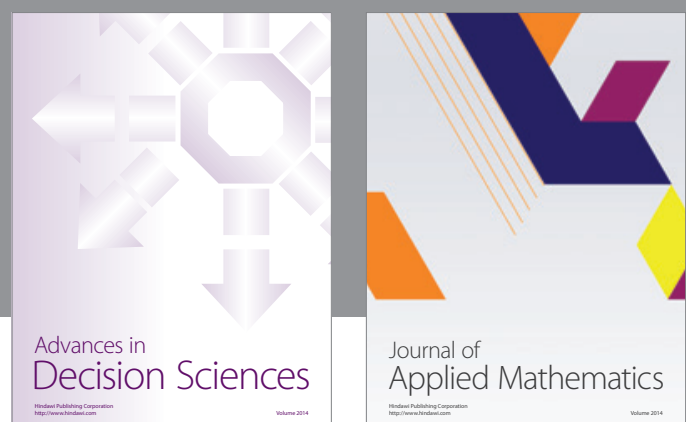

Journal of

Applied Mathematics
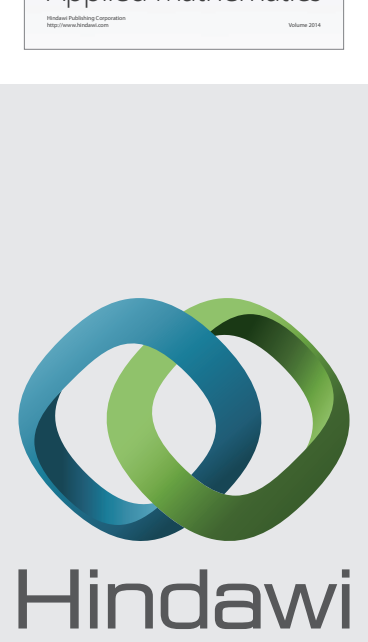

Submit your manuscripts at http://www.hindawi.com
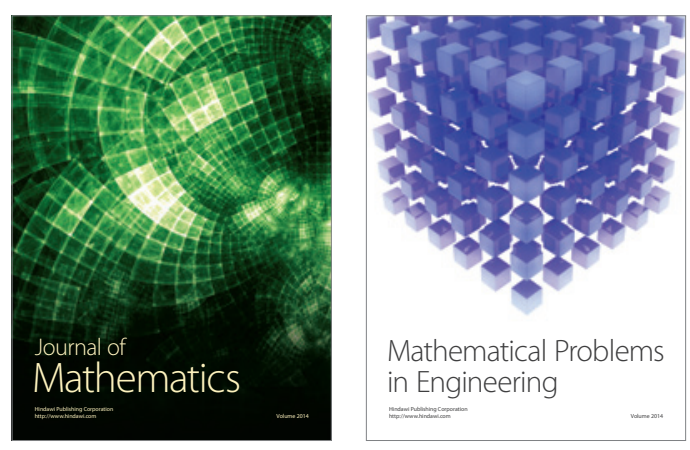

Mathematical Problems in Engineering
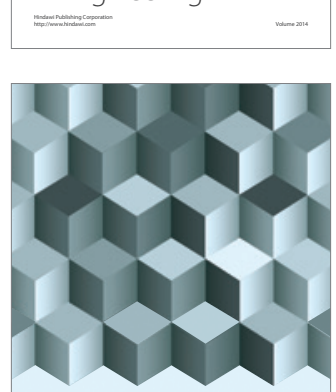

Journal of

Function Spaces
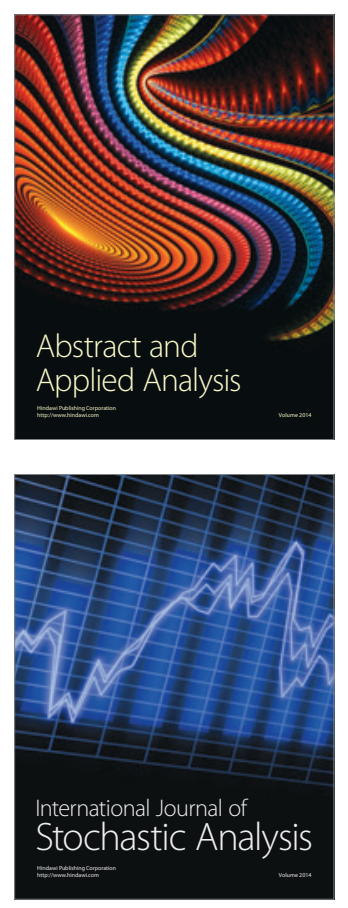

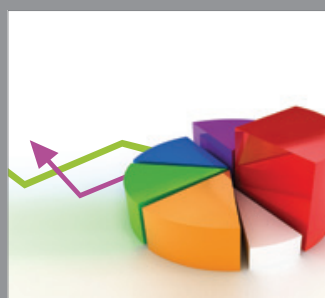

ournal of

Probability and Statistics

Promensencen
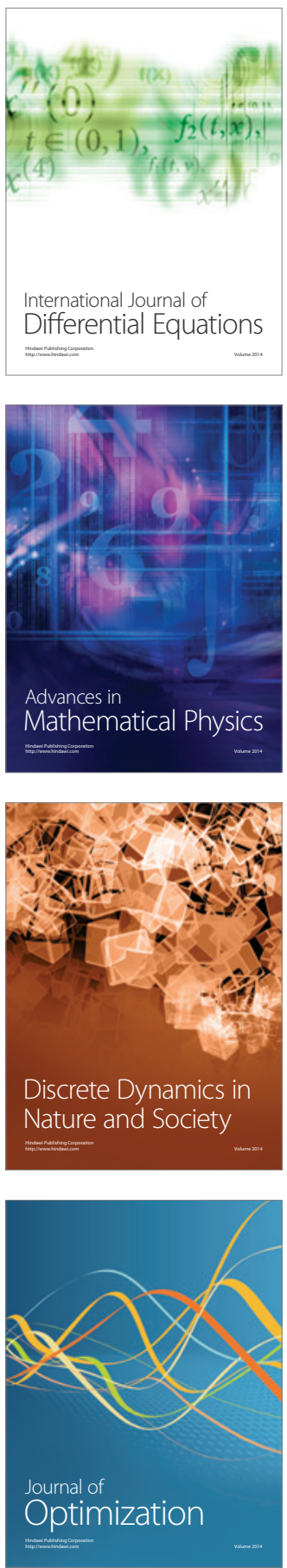